\title{
GLOMERULAR NUMBER AND CAPILLARY DIMENSIONS IN THE NORMAL LAMB KIDNEY
}

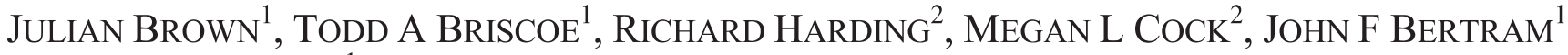 \\ AND JANE M BLACK ${ }^{1}$ \\ ${ }^{1}$ Departments of Anatomy \& Cell Biology and ${ }^{2}$ Physiology, Monash University, Victoria 3800, Australia \\ e-mail: jane.black@med.monash.edu.au \\ (Accepted October 10, 2002.)
}

\begin{abstract}
Reduced nephron endowment, either inherited or acquired, has been linked to adult renal pathology as well as hypertension. The sheep provides an excellent model for studying nephrogenesis and renal development because, as in humans, nephrogenesis is complete before birth. In the present study, the physical disector/fractionator method was used to estimate the total number of glomeruli, and thereby nephrons, in normal lambs. Glomerular capillary parameters including mean capillary length per glomerulus, mean capillary surface area per glomerulus and total renal filtration surface area (TRFSA) were also estimated. Total glomerular, and hence nephron number was 333,832 $\pm 69,560$ (mean \pm standard deviation). TRFSA was $10.95 \pm 3.64 \times 10^{4} \mathrm{~mm}^{2}$. These results establish a methodology for future investigations, using the sheep as a model, into the effects of depleted nephrogenesis on renal pathology in later life.
\end{abstract}

Keywords: glomeruli, lamb, nephron, renal filtration surface area, stereology.

\section{INTRODUCTION}

Hypertension has a long association with disorders of the kidney (Bright, 1836). The kidney is the ultimate regulator of blood pressure, and therefore invariably implicated in its pathogenesis (Guyton et al., 1972). It has been postulated that a major renal abnormality that initiates essential hypertension is decreased filtration surface area, due to either a reduced number of nephrons, or a decrease in glomerular filtration surface area per nephron. It is proposed that systemic hypertension then leads to glomerular capillary hypertension and eventual glomerular sclerosis, which in turn further decreases filtration surface area, perpetuating a vicious cycle (Brenner et al., 1988).

Kidney development in the foetal lamb mirrors closely that in human development (Gimonet et al., 1998). In the sheep, induction of the permanent kidney (metanephros) begins at day 30 of gestation (Wintour et al., 1996), and a centrifugal nephron developmental pattern is observed, with nephron induction first observed at about day 50. Nephrogenesis reaches its peak at day 80 , and as in humans, it is not a continuous phenomenon. No significant nephrogenesis is observed after 120 days (Gimonet et al., 1998), and after 140 days of gestation no further evidence of nephrogenesis is observed.
Quantitative morphological literature on ovine renal development is scarce. The aim of this study was to determine the total number of glomeruli, and hence nephrons in the normal lamb kidney. A second stereological exercise was also conducted on the same lamb kidneys to estimate mean capillary length per glomerulus, mean capillary surface area per glomerulus and total renal filtration surface area (TRFSA). These measures of postnatal ovine renal morphology may be relevant to understanding the role of foetal nephrogenesis in hypertension and renal pathology later in life.

\section{METHODS}

The following animal experiments were approved by the Monash University, Department of Physiology Ethics Committee and the treatment and care of the animals conformed to the Australian Code of Practice for the Care and Use of Animals for Scientific Purposes.

\section{ESTIMATION OF GLOMERULAR NUMBER}

\section{Tissue Preparation And Sampling}

The right kidneys of full term Border Leicester X Merino lambs of 8 weeks postnatal life were removed 
following administration of a lethal dose of pentabarbitone sodium $(16.25 \mathrm{mg} / \mathrm{kg})$. The kidneys were injected, via the exposed renal artery, with $0.1 \mathrm{~mL}$ papaverine hydrochloride $(1.2 \mathrm{mg})$ to achieve vascular dilation. The kidney vasculature was then flushed with saline to remove blood and subsequently perfusion-fixed with $4 \%$ paraformaldehyde plus $0.2 \%$ glutaraldehyde in $0.1 \mathrm{M}$ phosphate buffer at a $\mathrm{pH}$ in the range of 7.2 to 7.4. Following storage in fixative, each kidney was removed of its capsule and accompanying fat and sampled as illustrated in Fig. 1.

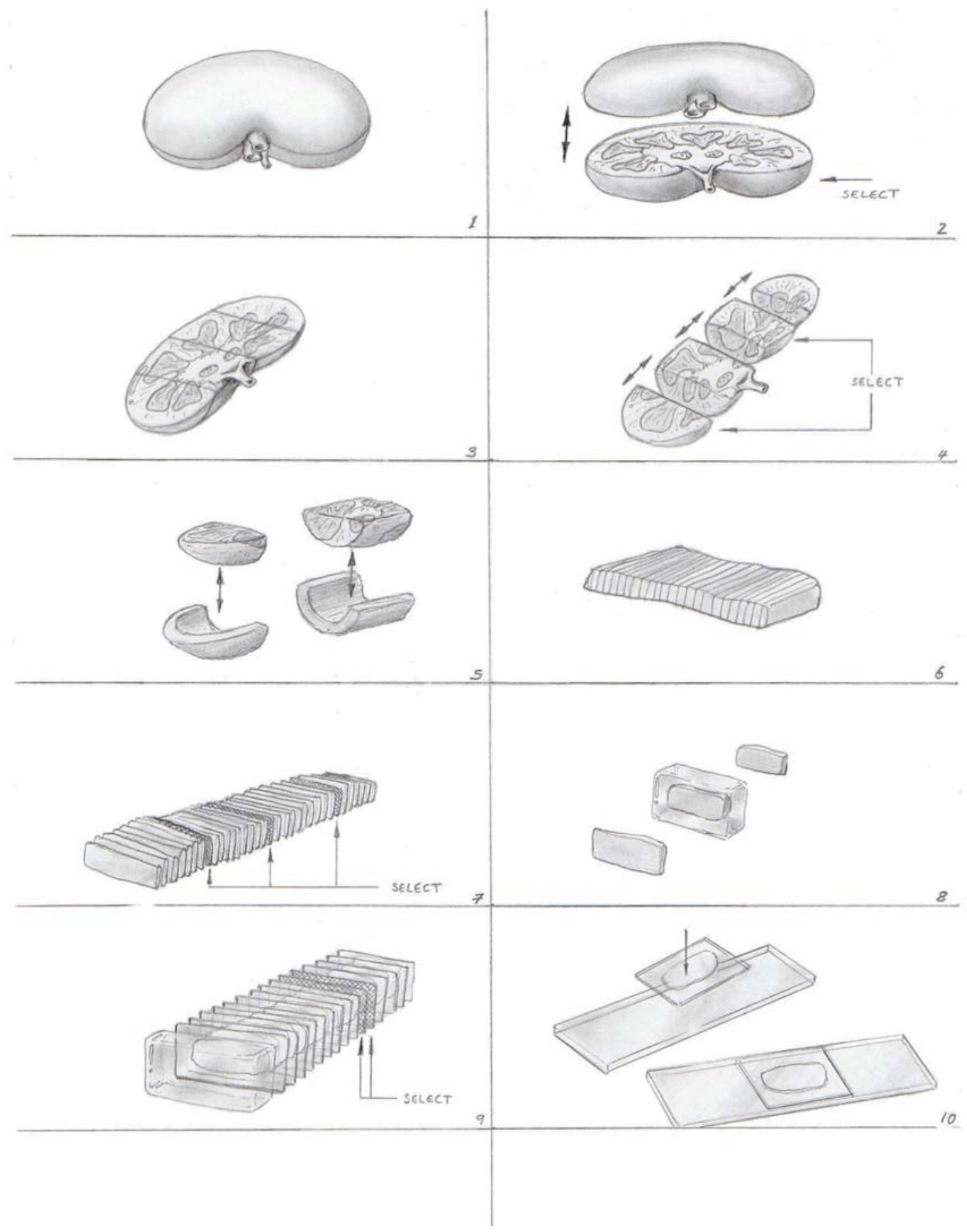

Fig. 1. Diagram illustrating the sequential sampling of the kidney. The whole kidney (1) was cut in half in the longitudinal plane (2). One half of the kidney was cut into four (3) and two of the four segments sampled (4). The cortex was carefully cut away from the medulla (5) and each piece sliced into $1.5 \mathrm{~mm}$ thick slices (6). Every $10^{\text {th }}$ slice was sampled (beginning with a random number between 1 and 10) (7) and embedded in glycolmethacrylate (8). The blocks were exhaustively sectioned at $20 \mu \mathrm{ms}$ and every $10^{\text {th }}$ and $11^{\text {th }}$ section (beginning with a random number) were sampled (9) and mounted onto slides (11). 
Each kidney was cut in half longitudinally through the hilus and one half chosen at random was stored. The other half was cut laterally into four segments, every second of which (starting randomly from the first or second segment) was sampled. Any remaining connective tissue was removed and the medulla cut away. Each segment was then cut into slices approximately $1.5 \mathrm{~mm}$ thick using a razor blade cutting device. An average of 100 slices were obtained per kidney. Every tenth slice (starting from the $n$th slice where $n$ is a random number between one and ten) was placed in cassettes, dehydrated through a series of graded alcohols, infiltrated and embedded in glycolmethacrylate Technovit 7100 (Heraeus Kulzer, Germany) blocks (approximately $14 \mathrm{~mm} \times 16 \mathrm{~mm} \times$ $5 \mathrm{~mm}$ ). Between eight and twelve slices were embedded for each kidney. To save time and resources, if slices were small enough, two or more would be embedded in the same block. These blocks were then mounted on a microtome chuck and the distance from the base of the chuck to the block face was recorded using a digital micrometer.

Using a Leica 2165 rotary microtome fitted with glass knives, blocks were exhaustively sectioned at a nominal thickness of $20 \mu \mathrm{m}$ and the number of sections obtained was recorded. The distance from the base of the chuck to the block face was remeasured using the digital micrometer and average section thickness for each block $(\bar{t})$ was estimated.

With the first chosen at random, every tenth "sampled section" and eleventh "look-up section" thereafter was mounted on to glass slides (together referred to as a "section pair"). Section pairs were then dried, stained with haematoxylin and eosin, and placed in a $40^{\circ} \mathrm{C}$ oven overnight.

\section{Estimating Total Glomerular Number $\left(\mathrm{N}_{\text {glom,kid }}\right)$}

The fraction of the sampled tissue $\left(\mathrm{P}_{\mathrm{s}}\right)$ was determined by counting grid points overlying tissue in a section projected on to a $20 \mathrm{~mm}$ point grid at a magnification of 24.25 times using a Fuji Minicopy Reader (hereinafter referred to as a 'microfiche'). The first section of each section pair was counted for each block of each kidney. Where two or more sections were contained on one slide, the sampled section was isolated and counted separately.

Glomeruli were counted using a physical disector technique (Nyengaard and Bendtsen, 1990). Only complete sections, that is, sections in which both the circumference and centre of the tissue were intact were used. Section pairs were projected side-by-side in a darkened room by two adjacent light microscopes (Olympus BX50F4) equipped for projection. Again, if there were two or more sections on one slide, the sampled section was isolated and counted separately. The first (sampled) section was placed on the stage of the right microscope and the second (look-up) section placed on the stage of the left microscope. The right microscope was equipped with an electronic motorised stage with an automated stepping action for systematic uniformly random sampling. The left microscope had a manual rotating stage to aid in alignment of the section. A final magnification of 300 times was used. An unbiased counting frame was applied to the field of vision for both microscopes $(60 \mathrm{~mm} 3 \times 3$ orthogonal grid). For each field of view ('frame'), the corresponding region of the sampled section was found on the look-up section and glomerular profiles sampled in the counting frame that were not present in the look-up section were counted as an "event" $Q^{-}$. To double efficiency, glomerular profiles in the lookup section but not in the sampled section were also counted. In accordance with the Gundersen criteria, (Gundersen 1977 and 1986), glomerular profiles that fell on the forbidden lines (the lower and left edges of the counting frame) were excluded, as were profiles that fell on an artificial edge of the tissue or otherwise incomplete, or whose position in the tissue was obviously disturbed by processing.

The area of tissue allowed for counting glomeruli $\left(\mathrm{P}_{\text {kid }}\right)$ was also counted simultaneously for every frame analysed above. A $30 \mathrm{~mm} 6 \times 6$ point grid was placed on top of the already superimposed $3 \times 3$ orthogonal grid, retaining the final magnification of 300 times. The number of grid points falling on the tissue was counted as $\mathrm{P}_{\text {kid }}$ and the number of points overlying glomeruli $\mathrm{P}_{\text {glom }}$ and renal corpuscles $\mathrm{P}_{\text {corp }}$ (used to estimate glomerular and renal corpuscle volumes) were also counted using the same grid and magnification. Only the first section of each pair was counted.

The total number of glomeruli in the kidney, $\mathrm{N}_{\text {glom,kid, }}$ was determined using:

$$
\mathrm{N}_{\text {glom,kid }}=\frac{1}{S F} \times \frac{\mathrm{P}_{\mathrm{s}} \times a(p) m}{\mathrm{P}_{\text {kid }} \times a(p) d} \times \frac{Q^{-}}{2},
$$

where $S F$ is the sampling fraction defined as the product of: (i) the sampling fraction of the sampled segments $\left(\frac{1}{4}\right)$; (ii) the sampling fraction of the sampled slices $\left(\frac{1}{10}\right)$; and (iii) the section sampling fraction for every tenth section taken $\left(\frac{1}{10}\right) \cdot \frac{P_{s} \mathrm{x} a(p) m}{P_{k i d} \mathrm{x} a(p) d}$ is the reciprocal of the fraction of the section areas or 
frames used for glomerular counting and $Q^{-}$was the number of glomerular events recorded. $Q^{-}$is divided by 2 because each pair was analysed for "events" in two directions. The area associated with each point of the microfiche grid, $a(p) m$, was calculated by dividing the length between points on the superimposed point grid used $(20 \mathrm{~mm})$ by the final magnification of the section $(24.25 \times)$ and then squaring the result to give an area of $0.6802 \mathrm{~mm}^{2}$. The area associated with each point on the physical disector grid, $a(p) d$, was similarly calculated by dividing the length between points $(30 \mathrm{~mm})$ by the final magnification achieved $(300 \times)$ and then squaring the result to give an area associated with each point of $0.01 \mathrm{~mm}^{2}$.

Between 30 and 80 frames that contained tissue per pair were analysed stepping at a constant distance of $1300 \mu \mathrm{m}$ in the $\mathrm{X}$ direction and $1300 \mu \mathrm{m}$ in the $\mathrm{Y}$ direction starting from a position randomly obtained. The variation in the number of frames analysed per pair was due to different sizes of the sections analysed. The number of frames analysed per pair does not affect the final result because the area of tissue analysed is taken as a fraction of the total tissue sampled, i.e. the fraction of the tissue viewed is the quotient of $\left(\mathrm{P}_{\mathrm{kid}} \times a(p) d\right)$ and $\left(\mathrm{P}_{\mathrm{s}} \times a(p) m\right)$, thus a higher $Q^{-}$resulting from analysing more frames will be compensated by a corresponding increase in $\mathrm{P}_{\text {kid }}$. For each kidney, between eight and twelve pairs of sections were analysed and $Q^{-}$was at least 100 .

The volume of the kidney $\left(\mathrm{V}_{\mathrm{kid}}\right)$ was calculated as being the product of the area of the sections $\left(\mathrm{P}_{\mathrm{s}} \times \mathrm{a}(p) m\right)$, average section thickness $(\bar{t})$ and the reciprocal of the sampling fraction $\left(\frac{1}{S F}\right)$.

$$
\mathrm{V}_{\mathrm{kid}}=\frac{1}{S F} \times \mathrm{P}_{\mathrm{s}} \times a(p) m \times \bar{t} .
$$

Mean glomerular tuft $\left(\overline{\mathrm{v}}_{\text {glom }}\right)$ and renal corpuscle $\left(\overline{\mathrm{V}}_{\text {corp }}\right)$ volumes were estimated by first determining volume densities $\mathrm{V}_{\mathrm{Vglom}, \mathrm{kid}} \mathrm{V}_{\mathrm{Vcorp}, \mathrm{kid}}$ and the number of glomeruli per unit volume of kidney $\mathrm{N}_{\mathrm{Vglom}, \mathrm{kid}} \mathrm{using}$ the following formulae (Bertram, 1995):

$$
\begin{aligned}
& \mathrm{V}_{\mathrm{V}_{\text {glom,kid }}}=\frac{\mathrm{P}_{\text {glom }}}{\mathrm{P}_{\text {kid }}}, \\
& \mathrm{V}_{\mathrm{V}_{\text {corp }, \text { kid }}}=\frac{\mathrm{P}_{\text {corp }}}{\mathrm{P}_{\text {kid }}},
\end{aligned}
$$

$$
\mathrm{N}_{\mathrm{V}_{\text {glom, kid }}}=\frac{\mathrm{N}_{\text {glom,kid }}}{\mathrm{V}_{\text {kid }}} \text {. }
$$

$\bar{v}_{\text {glom }}$ and $\bar{v}_{\text {corp }}$ were thus determined as the quotient of their volume densities with glomerular numerical density:

$$
\begin{gathered}
\overline{\mathrm{V}}_{\text {glom }}=\frac{\mathrm{V}_{\mathrm{V}_{\text {glom,kid }}}}{\mathrm{N}_{\mathrm{V}_{\text {glom,kid }}}}, \\
\overline{\mathrm{V}}_{\text {corp }}=\frac{\mathrm{V}_{\mathrm{V}_{\text {corp,kid }}}}{\mathrm{N}_{\mathrm{V}_{\text {glom,kid }}}} .
\end{gathered}
$$

\section{DETERMINATION OF GLOMERULAR CAPILLARY LENGTH AND SURFACE AREA}

\section{Tissue preparation and sampling}

In slices not designated for embedding in glycolmethacrylate, cubes of cortex extending from the outside capsule to the medulla were systematically sampled. These were placed in vials, dehydrated through a series of graded alcohols, infiltrated and embedded in epon-araldite. A total of eight samples from each kidney were embedded, one per block. Subsequently, these eight blocks were sectioned at a nominal thickness of $1 \mu \mathrm{m}$ using a Reichert UM-03 microtome and stained with toluidine blue. One section per block was projected on to a tabletop in a darkened room at a magnification of 150 times. Two glomeruli per section were sampled according to Nyengaard (1993). In brief, the number of glomerular profiles in the section was counted $(N)$ and each assigned a number. A random number was selected, $n_{1}$, and the corresponding glomerular profile was sampled. A second profile, $n_{2}$, was sampled using the formula $n_{2}=n_{1}+\frac{N}{2}$, rounding up. Where $n_{2}$ was larger than the total number of profiles in the section, $n_{2}$ was subtracted from the total number of profiles, and the corresponding glomerular profile was sampled. In total, two glomerular profiles from each block were sampled and a total of 16 glomerular profiles were sampled per kidney.

\section{Estimating glomerular capillary length and surface area}

The selected glomeruli were examined at a final magnification of 1500 times using an oil immersion lens, again projected on a flat surface in a darkened room. The corpuscle capsule and all capillary profiles in 
the glomerular tuft were traced on to A3 paper. A 20 $\mathrm{mm}$ orthogonal grid was placed over each glomerular profile and grid points falling within Bowman's capsule $\left(\mathrm{P}_{\text {corp }}\right)$, the number of capillary profiles $(Q)$, and the number of intersections between the vertical and horizontal grid lines ( $\mathrm{I}_{\text {cap }}$ ) and the luminal membrane of endothelial cells was counted. The area associated with each point on the orthogonal grid used, $a(p) d$, was calculated by dividing the length between points by the final magnification achieved and then squaring the result. Fig. 2 provides an illustration of this process.
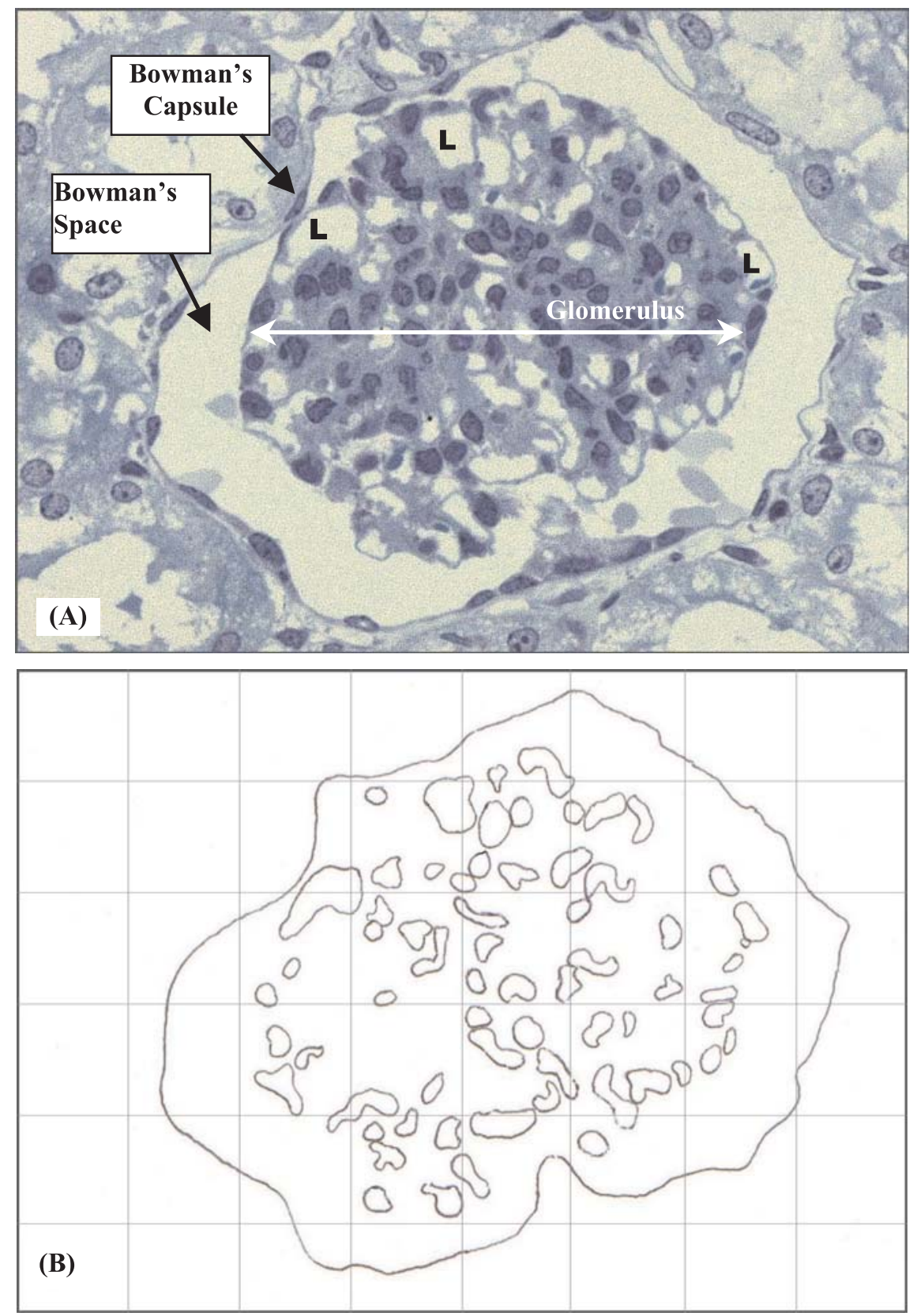

Fig. 2. (A) is a high-resolution image of a glomerular profile (from a $1 \mu \mathrm{m}$ section embedded in epon-araldite and stained with toluidine blue). Note: the renal corpuscle comprises of Bowman's capsule, Bowman's space and the glomerulus. The glomerulus is composed of a tuft of glomerular capillaries in a supporting mesangium. The areas marked with $L$ represent the lumens of capillary profiles that comprise the glomerular tuft. The glomerular capillaries are cut in transverse and oblique sections. (B) is a corresponding tracing of the same glomerulus showing capillary profiles and a superimposed orthogonal grid used for counting the number of capillary profiles, intersections of capillary walls with both horizontal and vertical lines and intersections of the grid overlying the renal corpuscle. 
The length density of the capillaries in the corpuscle $\left(\mathrm{L}_{\mathrm{V}_{\text {cap.corp }}}\right)$ was determined using the standard stereological formula (Bertram, 1995):

$$
\mathrm{L}_{\mathrm{V}_{\text {cap, corp }}}=\frac{2 Q}{\mathrm{P}_{\text {corp }} \mathrm{x} a(p) d} .
$$

The surface area density of capillaries in renal corpuscles $\left(\mathrm{S}_{\mathrm{V}_{\text {cap,corp }}}\right)$ was determined using the standard stereological formula (Bertram, 1995):

$$
\mathrm{S}_{\mathrm{V}_{\text {cap, corp }}}=\frac{2 \mathrm{I}_{\text {cap }}}{\mathrm{P}_{\text {corp }} \mathrm{x} \times d},
$$

where $k$ is 2 for the orthogonal grid and $d$ is the length of the grid line adjusted for magnification. These estimators rely on the reasonable assumption that the glomerular capillaries are isotropic orientated. The respective total capillary length $\overline{1}_{\text {cap,corp }}$ and surface area $\bar{S}_{\text {cap,corp }}$ per average glomerulus were calculated as the product of the above densities and $\overline{\mathrm{V}}_{\text {corp }}$ (Bertram, 1995):

$$
\begin{aligned}
& \overline{1}_{\text {cap,corp }}=\mathrm{L}_{\mathrm{V}_{\text {cap }, \text { corp }}} \times \overline{\mathrm{V}}_{\text {corp }}, \\
& \overline{\mathrm{S}}_{\text {cap,corp }}=\mathrm{S}_{\mathrm{V}_{\text {cap, corp }}} \times \overline{\mathrm{V}}_{\text {corp }} .
\end{aligned}
$$

Total renal filtration surface area TRFSA $\left(\mathrm{S}_{\text {cap,kid }}\right)$ was subsequently determined as the product of the mean capillary surface area per glomerulus $\overline{\mathbf{S}}_{\text {cap,corp }}$ and the total number of glomeruli in the kidney $\left(\mathrm{N}_{\text {glom,kid }}\right)$ :

$$
\mathrm{S}_{\text {cap }, \mathrm{kid}}=\overline{\mathrm{S}}_{\mathrm{cap}, \mathrm{corp}} \mathrm{XN}_{\text {glom,kid }} \text {. }
$$

\section{RESULTS}

The results are presented in Table 1. All values are expressed as mean \pm standard deviation (SD).

\section{DISCUSSION}

The method employed to estimate glomerular, and thereby nephron number, in the present study is based closely on the method employed by Johnson et al. (2000) to estimate nephron number in human kidneys, which was in turn based on Nyengaard and Bendtsen (1992). The total number of nephrons in the lamb kidney has been estimated in a number of earlier studies. Robillard et al. (1981) used a method, which they derived from McNay and Abe (1970) that was based on multiplying the number of glomeruli per unit mass of cortex by the volume of the cortex. Geometric assumptions were required to estimate cortical volume and it is not clear how glomerular numerical density was estimated. The estimates of nephron number reported in the newborn lamb $(n=8)$ and 130 day foetus $(n=9)$ by Robillard et al. (1981) $(433,704 \pm 21,533$ and $468,296 \pm 41,173$, respectively) are somewhat higher than the estimates obtained in the present study. Indeed, the estimate in the newborn lambs was $30 \%$ higher than the present estimate.

Table 1. Kidney volumes and light microscopic stereological estimates for 5 lambs. Note the inter-individual coefficient of variation (CV) is expressed as a percentage of the mean.

\begin{tabular}{llllllll}
\hline Animal & $\begin{array}{l}\text { Kidney } \\
\text { volume }\end{array}$ & $\begin{array}{l}\text { Total number } \\
\text { of glomeruli } \\
\text { per kidney }\end{array}$ & $\begin{array}{l}\text { Mean } \\
\text { glomerular tuft } \\
\text { volume }\end{array}$ & $\begin{array}{l}\text { Mean renal } \\
\text { corpuscle } \\
\text { volume }\end{array}$ & $\begin{array}{l}\text { Surface area of } \\
\text { capillaries in } \\
\text { corpuscle }\end{array}$ & $\begin{array}{l}\text { Length of } \\
\text { capillaries in } \\
\text { corpuscle }\end{array}$ & TRFSA \\
\cline { 2 - 7 } & $\mathrm{V}_{\text {kid }}$ & $\mathrm{N}_{\text {glom,kid }}$ & $\begin{array}{l}\mathrm{V}_{\text {glom }} \\
\mathrm{cm}^{3} \times 10^{-3}\end{array}$ & $\begin{array}{l}\overline{\mathrm{V}}_{\text {corp }} \\
\mathrm{mm}^{3} \times 10^{-3}\end{array}$ & $\begin{array}{l}\overline{\mathrm{S}}_{\text {cap,corp }} \\
\mathrm{mm}^{2}\end{array}$ & $\begin{array}{l}\overline{1}_{\text {cap,corp }} \\
\mathrm{mm}\end{array}$ & $\begin{array}{l}\mathrm{S}_{\text {cap,kid }} \\
\mathrm{mm}^{2} \times 10^{4}\end{array}$ \\
\hline & & & & & & & \\
1 & 36.3 & 397752 & 2.01 & 2.88 & 0.297 & 23.30 & 16.41 \\
2 & 22.7 & 301687 & 1.70 & 2.86 & 0.192 & 13.76 & 7.94 \\
3 & 25.6 & 258985 & 1.83 & 2.94 & 0.248 & 19.05 & 8.79 \\
4 & 20.9 & 293297 & 1.60 & 2.82 & 0.215 & 19.04 & 8.62 \\
5 & 38.7 & 417440 & 2.30 & 3.63 & 0.229 & 21.57 & 13.00 \\
& & & & & & & \\
Mean & 28.8 & 333832 & 1.88 & 3.02 & 0.240 & 19.34 & 10.95 \\
$\mathrm{SD}$ & 8.1 & 69560 & 0.27 & 0.34 & 0.040 & 3.60 & 3.64 \\
$\mathrm{CV}(\%)$ & 28.24 & 20.84 & 14.63 & 11.21 & 16.76 & 18.63 & 33.28 \\
\hline
\end{tabular}


More recently, Bains and Sibbons (1996) used a physical disector/Cavalieri combination to estimate glomerular number in the lamb kidney. Glomerular number in a control group $(n=6)$ was compared to an intra-uterine growth retarded (IUGR) group $(n=6)$. They found that the control group had an average of 289,439 nephrons per kidney whereas the IUGR group had an average of 189,117 nephrons per kidney (Bains and Sibbons, 1996). The estimates of Bains and Sibbons (1996) for the normal lamb are only $13 \%$ less than the present estimates. This is most likely due to differences in sheep strain or birth weight.

There were similarities in the method employed by Bains and Sibbons (1996) compared to the present study. However, some technical differences deserve mention. For example, the present study used glycolmethacrylate to embed slices of kidney, whereas Bains and Sibbons (1996) embedded their slices in paraffin wax, which is prone to large levels of tissue shrinkage. Schmitz et al. (1990) found that the volume of glomeruli embedded in glycolmethacrylate was approximately double that of glomeruli embedded in paraffin. Whilst shrinkage is not relevant for estimating nephron number, it is relevant for estimation of kidney volume, capillary length and surface area and TRFSA. The purpose of using glycolmethacrylate (as opposed to paraffin wax) was to reduce shrinkage of tissue subsequent to initial fixation as little as possible or alternatively to the same rate of shrinkage as epon-araldite (Bertram 1995). A potential disadvantage of this technique is that it is vulnerable to technical problems where environmental conditions may result in different levels of shrinkage between the epon-araldite and the glycolmethacrylate, needing the possible inclusion of a shrinkage correction. However environmental controls in laboratories are strictly monitored and controlled (including storage of reagents) to ensure consistent environmental conditions.

Further differences in protocol include the sections being cut $10 \mu \mathrm{m}$ apart compared to $20 \mu \mathrm{m}$ sections that were cut in the present study. Bains and Sibbons (1996) counted 40 frames per section pair whereas a variable number of frames per pair were counted in the present study. Counting in a fixed number of counting frames per section, as performed by Bains and Sibbons (1996) can lead to bias of a disector times Cavalieri design (depending on the heterogeneity of the observed tissue). It should be noted that counting a variable number of frames per section or changing the stepping length should not affect estimates for total nephron number using a physical disector/fractionator design. This is because the area of kidney viewed using the physical disector is accounted for by the use of $\frac{\mathrm{P}_{\mathrm{s}} \mathrm{x} a(p) m}{\mathrm{P}_{\mathrm{kid}} \mathrm{x} a(p) d}$; this is an advantage because this caters for significant biological variation between specimens.

An important advantage of the physical disector/fractionator combination is that glomeruli are counted in a known and predetermined fraction of the kidney. No assumptions need be made about glomerular geometry, and there is no need to determine section thickness (Bertram et al. 1992). However, an ongoing requirement as noted by Nyengaard and Bendtsen (1990 and 1992) and Bertram et al. (1992) is consistent and unambiguous identification of glomeruli. Strict glomerular identification criteria are important, with identification assisted by the quality of the staining and the choice of objective lens, and hence magnification, of the image observed.

The criteria used in the present study for identifying glomerular profiles was a collection of cells that belonged to an obvious glomerular tuft, confirmed by the presence of either Bowman's capsule or space, as distinguished by the stain. To further maintain consistency, glomerular profiles in the sampled section were identified as such without reference to the look-up section and vice versa. If the look-up section was constantly used to confirm the presence of a glomerular profile in the sampled section, this would mean that where the glomerular profile was absent from the look-up section, this may lead the observer to the erroneous conclusion that the cells in the sampled section did not form part of the glomerular profile and thus an "event" would be missed.

The present study has found that comprehensive training is required to use the physical disector/ fractionator technique. If the stereology is correctly performed and consistent criteria are used, this should not lead to significant differences between studies. Where different observers consistently count more or less than the other, this indicates that at least one of the observers is not applying the stereological methods correctly and hence needs further training. It is also vital to have a continued program of quality control to ensure observers do not change the way they count as a large project continues.

As noted above, glomerular profiles were sampled according to the criteria of Gundersen 1977 and 1986: (1) glomerular profiles were only sampled when the profile was contained fully within the 'forbidden lines' and (2) glomeruli were only sampled where there was no artificial surface within the corresponding frame. Consistent with this, any 
glomeruli that fell on any artificial surfaces (surfaces that were produced by the knife or razor blade cutting device during initial sampling, or later by the microtome) or were otherwise incomplete were excluded in accordance with the glomerular identification criteria. Number fractions were used instead of weight fractions when calculating the sampling fraction $(S F)$. Whilst it is noted that manually cutting a specimen in half will invariably sample more or less than $50 \%$ of the whole, it is our experience that weighing tissue stored in fixative that may not be $100 \%$ dry and continually evaporating is prone to gross error, especially when progressively weighing smaller pieces. Thus, number fractions were used to eliminate potential source of bias, although consequently there is less precision in the results obtained.

The current estimate of total nephron number in the lamb kidney is in good agreement with the estimate reported for the normal lamb kidney at approximately 130 days gestation by Douglas-Denton et al. (2002). Using the same method as the current study, Douglas-Denton et al. (2002) estimated that average total nephron number to be 365,672 just $9 \%$ greater than the current estimate.

The estimates of glomerular capillary length and surface area have coefficients of variation of between $16 \%$ and $19 \%$. Glomerular profiles sampled represented a good distribution of glomeruli throughout the cortex of each kidney. It is thus considered that the results from the control group are true of the average lamb and that studies looking into effects of prematurity on glomerular capillary structure in the sheep can rely on these results in comparison studies.

\section{ACKNOWLEDGEMENTS}

This study was supported by a grant from the Australian National Health and Medical Research Council (NH\&MRC). The authors gratefully acknowledge the assistance of Mark Ting in preparing and editing illustrations.

\section{REFERENCES}

Bains RK, Sibbons PD (1996). Stereological estimation of the absolute number of glomeruli in the kidneys of lambs. Res Vet Sci 60:122-5.

Bertram JF (1995). Analysing renal glomeruli with the new stereology. Int Rev Cytol 161:111-72.

Bertram JF, Soosaipillai MC, Ricardo SD, Ryan GB (1992). Total numbers of glomeruli and individual glomerular cell types in the normal rat kidney. Cell Tissue Res 270:27-45.
Brenner BM, Garcia DL, Anderson S (1988). Glomeruli and blood pressure: Less of one, more the other? Am J Hypertens 1:335-7.

Bright R (1836). Tabular view of morbid appearance in 100 cases connected with albuminous urine. Guy's Hosp Rep 1:380-410.

Douglas-Denton R, Moritz KM, Bertram JF, Wintour EM (2002). Compensatory renal growth after unilateral nephrectomy in the ovine fetus. J Am Soc Nephrol 13:406-10.

Gimonet V, Bussieres L, Medjebeur AA, Gasser B, Lelongt B, Laborde K (1998). Nephrogenesis and angiotensin II receptor sub-types gene expression in the fetal lamb. Am J Physiol 274 (Renal Physiol 43):F1062-9.

Gundersen HJG (1977). Notes on the estimation of the numerical density of arbitrary profiles: the edge effect. J Microsc 111:219-23.

Gundersen HJG (1986). Stereology of arbitrary particles: A review of unbiased number and size estimators and the presentation of some new ones. J Microsc 143:3-45.

Guyton AC Coleman TG, Cowley AVJ, Scheel KW, Manning RDJ, Norman RAJ (1972). Arterial pressure regulation. Overriding dominance of the kidneys in long-term regulation and in hypertension. Am J Med 52:584-94.

Johnson KJ, Wreford NG, Hoy WE, Bertram JF (2000). Estimating total glomerular number in human kidneys with a physical disector/fractionator combination. Image Anal Stereol 20:105-108.

McNay JL, Abe Y (1970). Pressure dependant heterogeneity of renal cortical blood flow in dogs. Circ Res 27:571.

Nyengaard JR, Bendtsen TF (1990). A practical method to count the number of glomeruli in the kidney as exemplified in various animal species. Acta Stereol 9(2):243-58.

Nyengaard JR, Bendtsen TF (1992). Glomerular number and size in relation to age, kidney weight, and body surface in normal man. Anat Rec 232:194-201.

Nyengaard JR (1993). The qualitative development of glomerular capillaries in rats with special reference to unbiased stereological estimates of their number and sizes. Microvasc Res 45:243-61.

Robillard JE, Weismann DN, Herin P (1981). Ontogeny of single glomerular perfusion rate in fetal and newborn lambs. Pediatr Res 15:1248-55.

Schmitz A, Nyengaard JR, Bendtsen TF (1990). Glomerular volume in type 2 (Non-Insulin Dependant Diabetes) diabetes estimated by a direct and unbiased stereological method. Lab Invest 62:108-13.

Wintour EM, Alcorn D, Butkus A, Congiu M, Earnest L, Pompolo S, Polocnik SJ (1996). Ontogeny of hormonal and excretory function of the meso- and metanephros in the ovine fetus. Kidney Int 50:1624-33. 